addition, UC Davis students gained exposure to a scholar (Dr. Ellis) who works in a different educational context than they experience at Davis, thus enhancing their understanding of the range of potential career paths in the field. All students learned to work collaboratively and cooperatively to conduct the research, to develop technical and quantitative skills, and to advance psychological research.

There are several challenges to developing a program like ours. The first is to identify an appropriate research context. We have been effective because we have a long-standing ongoing collaboration. Thus, although student projects were self-contained, they fit into a broader research context. This has allowed us to combine student work with other work for the purposes of publication, and it has allowed us to fully engage in the research at both institutions. The second challenge is to identify appropriate student projects. Although our collaboration provided a context for student projects, it can also inhibit students' exploration of diverse topics. Because our work is often tied to federal funding, student projects must address the aims outlined in those grant-funded projects. This can provide students insight into how research is supported, and about the constraints placed on the research process by external funding. However, because of these constraints, we have worked with students to come up with creative solutions for them to be able to insert their own ideas into a project that fits within the scope of our ongoing work. Finally, our success depends on having laboratories that are identically equipped. Even slight changes in equipment or software would mean that data from our two labs could not be combined. We constantly are vigilant to maintain laboratory equivalence.

In summary, our collaboration has created high-impact learning environments that develop undergraduates' intellectual curiosity, ethical sensitivity, independence, and selfconfidence in the context of conducting authentic research. All Grinnell College students who have participated in our collaborative research currently are enrolled at Grinnell, are in formal research or fellowship positions, or are pursuing or have earned advanced degrees. Experiences in our laboratories set our students on a course for more challenging work and have served to positively shape their career trajectories. Q

doi: $10.18833 /$ curq/36/4/20

\section{Spreading High-Impact Practices from the Genomics Lab to the Entire College and Beyond}

Gita Bangera, Cheryl Vermilyea,

Bellevue College, gbangera@bellevuecollege.edu

Kim Harrington,

Tacoma Community College

In the 21st century, community colleges are emerging as the backbone of higher education in the United States. Nearly half of all undergraduates start their education at community colleges and the percentage is even higher for students from groups underrepresented in higher education. National Science Foundation (NSF) grants are valuable for developing high-impact practices by individual faculty at various institutions, including collaborations between community colleges and research institutions. However, these practices will not change the higher education landscape unless they are scaled up to spread across the institution and across multiple institutions. Here we describe our ongoing endeavor to expand a model from two NSF-funded programs and to identify critical components and strategies in this process.

The Community College Genomics Research Initiative (ComGen), an NSF-funded program, started as a collaboration between Bellevue College and the Root Disease and Biological Control Research Unit of the United States Department of Agriculture's Agriculture Research Service. The USDA scientists provided a small fragment DNA library, technical advice, and classroom visits. Students at Bellevue College (from firstyear to those with PhDs) enrolled in a stand-alone research course modeled as a one-quarter, graduate school experience. Without lectures or textbooks, students got a first-hand experience in being a scientist, including conducting original research in genomics and analyzing primary literature. The ComGen model focuses on a "hands-on, hands-off" method: hands-on for students and hands-off for the faculty. This method encourages faculty to empower students to experience the ambiguity, failure, and recovery from failure that are inherent in research. The ComGen program has drawn national attention, being featured in Science (McCook 2011) and drawing an invitation from the White House to engage in a pledge to increase the number of students in science, technology, engineering, and math (STEM) fields.

While the stand-alone research course had substantial impact, the number of students reached was limited since the course was an elective that many students could not fit into their schedules or afford. To expand the impact of the ComGen pedagogy, we introduced critical components of the original course into the cell biology course for biology 
majors at Bellevue College and Tacoma Community College (Tacoma CC). This strategy allowed significantly expanded numbers of students to participate in undergraduate research early in their studies. Our second NSF grant is currently taking this experience to students across the state of Washington. Bringing undergraduate research into the regular curriculum in this fashion has the potential to impact the diversity of the research establishment (Bangera and Brownell 2014).

To expand incorporation of research practices into curricula within our institutions, we have followed two different paths based on institutional culture. At Bellevue College, we have leveraged the success of ComGen into the founding of a new division called the RISE (Research, Innovation, Service and Experiential) Learning Institute, directed by a new dean of undergraduate research. The RISE Learning Institute will take innovative research and elements of the experiential-learning curriculum to departments across campus, not just those in STEM fields. The success of the original ComGen collaboration is helping to introduce these high-impact practices more broadly at Bellevue College.

At Tacoma CC, transforming the culture started with the incorporation of ComGen elements into the curriculum of the biology major, followed by incorporation of these elements into multiple other courses developed by the faculty. The success of these courses was leveraged by faculty into a request for funding to reenergize the science and engineering department and to integrate research and technology into courses, to increase mentoring in engineering, and to institute a divisional retreat to discuss best practices.

Both Bellevue College and Tacoma CC have benefited from the vision of their leadership, including their presidents and boards of trustees. Bellevue funded the purchase of a $\$ 60,000$ DNA sequencer and provided dedicated research space for ComGen activities. This commitment has now expanded through the development of the RISE Learning Institute, including funds for the salary of the dean of undergraduate research and a $\$ 5$-million remodeling of space to build a trans-disciplinary undergraduate research lab. Additional support has come through development of a "collaboratory" to showcase the process of research to all students and partial funding of a research lab technician. The Tacoma CC administration has approved reduced class sizes for research courses, provided release time for faculty to focus on incorporating research into the curriculum, and funded equipment and consumables. The college is now exploring the possibility of incorporating funding for research in both STEM and non-STEM areas into the operations of the college.

With our second NSF grant, we were able to train faculty from 18 different institutions (15 community colleges and three universities) in using research as a pedagogical tool. This rapid extension to almost half the community colleges in Washington state has been possible because the ComGen team has developed an easy-to-adopt model that allows faculty to embed this pedagogy into their existing curricula. To extend engagement to other colleges, the ComGen team has developed a comprehensive strategy that includes outreach to faculty, technicians, administrators, and staff. In addition to interested faculty, technicians are also invited to an annual summer workshop that covers the critical elements of the ComGen philosophy. To encourage interest and investment by other stakeholders, the team made presentations at venues such as the annual meetings of the vice presidents of instruction and of the non-academic staff of all 32 community colleges in the state.

Our experiences indicate that taking research to students in the context of their regular coursework is important, as is making adoption of the research pedagogy easy for faculty. Thus, building research into the normal functioning of the college is essential to bringing a culture of research to a community college. Until research becomes as much part of the culture at all community colleges as it is at Bellevue College or Tacoma CC, funding from NSF and other sources to develop and support communities of practice is critical.

\section{References}

Bangera, Gita, and Sara E. Brownell. 2014. "Course-Based Undergraduate Research Experiences Can Make Scientific Research More Inclusive." CBE-Life Sciences Education 13 (4): 602-606. doi: 10.1187/cbe.14-06-0099.

McCook Allison. 2011. "Two-Year Colleges Are Jumping Into the U.S. Research Pool." Science 333 (6049): 1572-73. doi: 10.1126/ science.333.6049.1572.

doi: $10.18833 /$ curq/36/4/21 